\title{
Mathematical modelling of electrode systems of electrostatic filtration processes
}

\author{
Leonid Ivanovich Koltunov ${ }^{1, *}$, and Alexander Stepanovich Kizhuk ${ }^{1}$ \\ ${ }^{1}$ Department of Engineering Cybernetics, Belgorod State Technological University n.a. V.G. Shukhov, Kostyukov Str., 46, 308012, \\ Belgorod, Russia
}

\begin{abstract}
The up-to-date air filtration and ionization systems and units for premises, buildings and constructions have been analyzed. The peculiarities of mathematical modeling with the results of numerical and experimental research of electrostatic systems and units, distributed and autonomous, are presented. The peculiarities of discrete models of objects under research in the operator form are given. The computing algorithms, the numerical modeling software environment structure and the functioning peculiarities of automatic control local systems of electrostatic objects as part of automated dispatch control system have been developed.
\end{abstract}

\section{Introduction}

The modern electrostatic systems and units, dealing with electrostatic purification, ionization and ozonation of air, are used at rocket launching systems, submarines, in hospitals, health resorts and other facilities; they are also embedded in car fascia panels, used in refrigerators, LCdisplays, air humidifying devices etc. It should be noted that these electrostatic systems provide the relatively high quality of air at the lowest energy consumption, unlike ventilation and air conditioning systems, in which the issues of energy saving and providing the high quality of air usually contradict each other. In this regard the development of electrostatic systems in conditions of energy saving is a topical problem [1].

The analysis of the modern development of electrostatic systems has shown that it develops in several ways. The development of the first way is connected with the name of professor A.L. Chizhevsky. He has designed a facility for generating negative air ions by means of creating high electrical voltages on the «needle-shaped» electrodes. The main peculiarity of these facilities is the application of negative electrodes (e.g., ionizer of «Aeroion-25» type). There also appear air ionizers to provide the balance of positive and negative air ions (e.g., the «Yantar» ionizer).

The second way, dealing with two-stage electrostatic precipitators for ventilation and air conditioning systems, was developed in the former USSR in 60-ies. But the main boost for the development of these electric precipitators was given by the company «Plymoth» (Sweden) in the sphere of purifying air from welding, oil and other finely-dispersed aerosols in the industrial conditions. Electric precipitators of the similar type (LLC «Elstat», Moscow) are used also for the purification of the intake air in ventilation systems in buildings of various purposes. The main peculiarity of these facilities is the application of two chambers: ionization chamber for the particles charging and precipitation chamber for catching them.

The third way appeared at the turn of the century almost simultaneously in a few developed countries, in a form of ionizing air cleaner for various spheres of application: for personal use (e.g., model XJ-888 of the company AirComfort, Italy); for transport (e.g., model CP-3 of the company Chung Pung, South Korea); for residential buildings and offices (e.g., models CVN63CX of the company Sharp, Japan and XJ 3500 of the company AirComfort, Italy); for industrial enterprises (model Eagle 5000 of the American company «EcoQuest International»); for embedding into ventilation and air conditioning systems (model DuctwoRx of the company «EcoQuest International») etc. The peculiarity of these facilities consists in creating the air environment with the required balance of fast positive and negative air ions in the premises of buildings and constructions, and the conditions for the air self-purification in a certain area of premises as well as its purification by filtering through this unit. The development of such appliances is essential at the present stage, as it deals with the protection of environment and the human health [2].

\section{Analysis of existing methods and models}

The analysis of the existing methods and models of electrostatic units research has demonstrated that analytical methods are not fit for determining the electrical potentials distribution field in the interelectrode systems of these appliances. At present, there are still no analytical expressions for the modern electric precipitator systems, taking into account their

\footnotetext{
* Corresponding author: koltunov 1 i@mail.ru
} 
appearance in the 60-ies of the last century, and as for the rapidly developing new trend of autonomous filtration systems with the simultaneous air ionization, it is developing almost empirically. Studying these units on the basis of computational experiments and numerical methods is the only way of obtaining relatively accurate results. Among the main methods of solving boundary value problems we should single out the finite difference method, which has a number of applications for the numerical study of electrostatic units with account of their structural features [3] (for example, flat polarizing electrodes with corona wire electrodes).

The purpose of this work is developing the basis for determining the characteristic regularities of electrostatic units for air filtration and ionization, and improving their efficiency with account of a wide range of structural features on the basis of integrated research with the use of up-to-date mathematical modeling and computational experiment technologies.

There is a lot of standard software for solving boundary value problems with differential equations in partial derivatives on the basis of using various numerical methods (MatLab, ELCUT, FemLab, ROSTWERK etc.). The usage of standard software is limited considerably for solving the boundary value problems, dealing with studying electrostatic units for air filtration and ionization, but there is the «IMPULS» modeling program, registered in the Russian Agency for software and database legal protection and aimed at calculating breakdown paths in electro-hydraulic units' interelectrode systems. On its base, with account of modernization, the electrostatic units can be studies with the subsequent creation of the software environment for the numerical modeling of such units.

The analysis of filtration processes' features in electrostatic units has demonstrated that they have both ionization areas for the particles charging, and electrostatic precipitation areas, where the unipolar corona can be also used. In some types of air cleaners the corona appears simultaneously on corona-forming electrodes in different parts of these units and at various high-voltage potentials on them. On the basis of analyzing the structures of electrostatic units for air filtration and ionization the basic standard layouts for their modeling have been designed.

For example, one of the modeling diagram for multichamber air cleaners with account of symmetry is shown in Fig. 1, $\left(\mathrm{G}_{\mathrm{i}}\right.$ - researched area boundaries $\mathrm{B}_{3}(\mathrm{x}, \mathrm{y}) ; \mathrm{K}_{1}$ and $\mathrm{G}_{6}$ - corona-forming electrodes, the dimensional ratio is determined with the type of various electrode systems of electric precipitators).

$G_{1}$

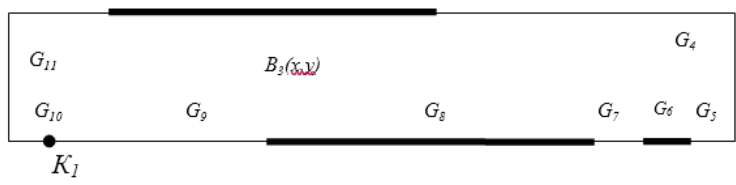

Fig. 1. The modeling diagram for multichamber air cleaners.
By means of studying the electrostatic units' electric fields a generic mathematical model is selected and substantiated, for which the boundary value problems with mixed-type boundary conditions, taking into account the basic limitations of the researched objects and the design features of these units, are formulated. The basis of the model is the approach, at which there is no need to determine the nonlinear dependence between the current density and the electric field intensity in the interelectrode systems of these objects [4]. The problem of calculating the electric field of electrostatic units' interelectrode space consists in determining the electric potential field $\varphi$ and the electrical function of the flux $\chi$, on basis of which the required parameters are calculated (electrostatic field intensity, effective ionization area etc.) with the subsequent determining of the range of appropriate rations of these objects' parameters.

For the boundary value problems of electrostatic units the conditions of finite difference method adaptation and transition to the finite-difference approximation of elliptical equations and boundary conditions are fulfilled. At this the peculiarities of the research objects' discrete models in the operator form are presented.

1. For example, for a boundary value problem (Fig. 1) with account of the second-order elliptical equation and the mixed-type boundary conditions the initial equations in operator form to determine the electric potential field $\varphi$ in node $(i, j)$ of the discrete area $\mathrm{B}_{\mathrm{g} 3}(\mathrm{x}, \mathrm{y})$ with the boundaries $\mathrm{Gi}$ are as follows.

$$
\Omega_{h} \varphi^{(h)} \equiv\left\{\begin{array}{l}
\Omega_{x x} \varphi_{m n}+\Omega_{y y} \varphi_{m n}=0,\left(x_{m}, y_{n}\right) \in B_{g 3}(x, y), \\
\varphi_{m n}=c_{1},\left(x_{m}, y_{n}\right) \in \boldsymbol{K}_{1}, \\
\varphi_{m n}=c_{2},\left(x_{m}, y_{n}\right) \in \boldsymbol{G}_{s}, \\
\varphi_{m n}=c_{3},\left(x_{m}, y_{n}\right) \in \boldsymbol{G}_{\boldsymbol{6}}, \\
\varphi_{m n}=0,\left(x_{m}, y_{n}\right) \in \boldsymbol{G}_{2}, \\
\Omega_{n} \varphi_{m n}=0,\left(x_{m}, y_{n}\right) \in \boldsymbol{G}_{1}, \boldsymbol{G}_{3}, \boldsymbol{G}_{4}, \boldsymbol{G}_{11}, \\
\Omega_{n} \varphi_{m n}=0,\left(x_{m}, \boldsymbol{y}_{n}\right) \in \boldsymbol{G}_{5}, \boldsymbol{G}_{7}, \boldsymbol{G}_{9}, \boldsymbol{G}_{10} .
\end{array}\right.
$$

Here $\Omega_{x x}=\partial^{2} / \partial x^{2} ; \quad \Omega_{y y}=\partial^{2} / \partial y^{2} ; \quad \Omega_{n}=\partial / \partial n$. The constants $\mathrm{c}_{\mathrm{i}}$ are determined by the values of high-voltage potentials on electrodes $\mathrm{K}_{1}, \mathrm{G}_{6}, \mathrm{G}_{8}$ with account of standard industrial prototypes of these objects.

2. The problem of determining the electrical function field of the flux $\chi$ for an electrostatic unit (Fig. 1) is the following. The function $\chi$ in node $(i, j)$ of the discrete area $B_{g 3}(x, y)$ with the boundaries $G_{i}$ is determined with account of the second-order elliptical equation and the mixed-type boundary conditions, which in operator form are as follows:

$$
\Omega_{h} \chi^{(h)} \equiv\left\{\begin{array}{l}
\Omega_{x x} \chi_{m n}+\Omega_{y y} \chi_{m n}=0,\left(x_{m}, y_{n}\right) \in B_{g 3}(x, y) \\
\Omega_{n} \chi_{m n}=0,\left(x_{m}, y_{n}\right) \in K_{1}, G_{2}, G_{6}, G_{s}, \\
\chi_{m n}=c_{1},\left(x_{m}, y_{n}\right) \in G_{1}, G_{10}, G_{11} \\
\chi_{m n}=c_{2},\left(x_{m}, y_{n}\right) \in G_{9} \\
\chi_{m n}=c_{3},\left(x_{m}, y_{n}\right) \in G_{7} \\
\chi_{m n}=0,\left(x_{m}, y_{n}\right) \in G_{3}, G_{4}, G_{5}
\end{array}\right.
$$


The mathematical models are intended for studying the characteristic regularities of electrostatic units for air filtration and ionization processes, and for determining the peculiarities of electrostatic precipitators and autonomous ionizing air cleaners with the purpose of improving their efficiency. The developed base allows researching the existing design solutions based on empirical approach at designing these appliances. So, the basis for the integrated research of electrostatic units with the use of up-to-date mathematical modeling technologies is presented [5].

The calculation data of the electrostatic units' interelectrode systems and of the experimental research are presented on a per-unit basis with account of dimension and similarity laws in relation to the objects' basic parameters. So, without the alteration of ionization chamber parameters the maximum value of the effective ionization area $\left(\mathrm{F}_{\mathrm{e}}^{*}\right)$ depends on the location of coronaforming electrode and the gap between the electrostatic precipitator's chambers. At reducing the gap $\delta$ from 30 $\mathrm{mm}$ to the minimum value the effective value $\mathrm{F}^{*} \mathrm{e}$ increases from the value $\mathrm{F}^{*}{ }_{\mathrm{e}(\min )}$ to the maximum $\mathrm{F}^{*}{ }_{\mathrm{e}(\max )}$ within the range from 1 to $\mathrm{m}_{11}=1,43$ $\left(\mathrm{m}_{11}=\mathrm{F}_{\mathrm{e}(\max )}^{*} / \mathrm{F}^{*} \mathrm{e}(\min )\right)$, whereas the maximum value $\mathrm{F}^{*}{ }_{\mathrm{e}(\max )}$ increases by 1,25 times. With account of the alteration of the electrode length $\left(\mathrm{L}^{*}{ }_{1}\right)$ in electrostatic precipitators' ionization chamber the maximum values of the effective ionization area increases by 2,5 times (Fig. 2). Through the research one of the important regularities of electrostatic units was revealed; it was determined that the increase of effective ionization area $\mathrm{F}^{*} \mathrm{e}$ of electrostatic precipitators is connected, firstly, with the ionization chamber's fringe effects, which move closer at its deminishing, which results in the considerable increase of $\mathrm{F}^{*} \mathrm{e}$ (by several times), and secondly, with the chamber's fields superposition.

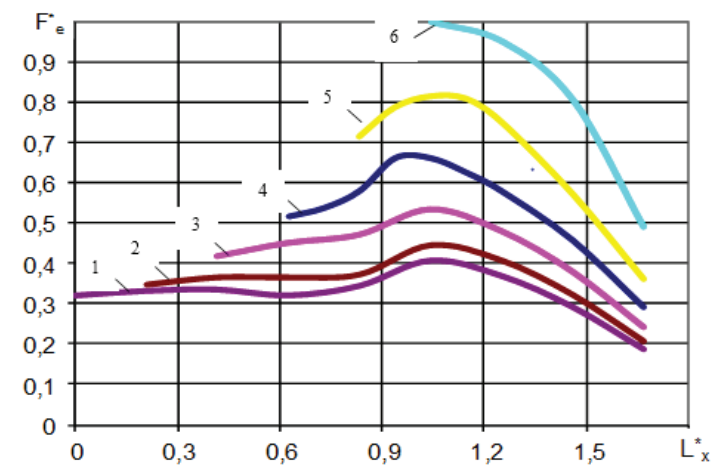

Fig. 2. Functional dependences $\mathrm{F}^{*}{ }_{\mathrm{e}}=f\left(\mathrm{~L}^{*}{ }_{\mathrm{x}}\right)$ with account of the alteration $\mathrm{L}^{*}{ }_{1}(\delta=$ const $): 1-\mathrm{L}^{*}{ }_{1}=1,625 ; 2-\mathrm{L}^{*}{ }_{1}=1,458 ; 3-$ $\mathrm{L}^{*}{ }_{1}=1,25 ; 4-\mathrm{L}^{*}{ }_{1}=1,042 ; 5-\mathrm{L}^{*}{ }_{1}=0,833 ; 6-\mathrm{L}^{*}{ }_{1}=0,625$.

To take into account the important features of electrostatic units the computer modeling methods have been developed, which allows determining their rational parameters with the possibility of creating the maximum effective ionization area and with account of air-flow rate in the interelectrode systems of these appliances. With account of the tendency of the consumed energy resources' continuous cost increase, the quasioptimal dimensions of these appliances allow improving their efficiency considerably.

At the designing stage of the single-chamber ionizing air cleaners' interelectrode systems it's necessary to take into account the possibility of improving their efficiency by means of selecting the appropriate ratios of their chambers' dimensions and with account of the coronaforming electrode location. For example, for ionizing air cleaners with the air consumption in the form of «ionic wind» at the chamber size alteration from $\mathrm{L}_{1}^{*}=1,625$ to $\mathrm{L}^{*}{ }_{1}=0,625$ the maximum value $\mathrm{F}^{*}{ }_{\mathrm{e}(\max )}$ increases by approximately 3 times (Fig. 3).

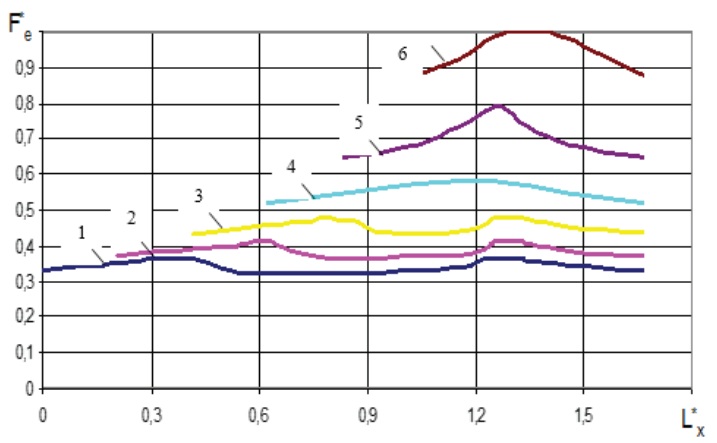

Fig. 3. Functional dependences $\mathrm{F}^{*}{ }_{\mathrm{e}}=f\left(\mathrm{~L}^{*}{ }_{\mathrm{x}}\right)$ with account of the alteration $\mathrm{L}^{*}{ }_{1}: 1-\mathrm{L}^{*}{ }_{1}=1,625 ; 2-\mathrm{L}^{*}{ }_{1}=1,458 ; 3-\mathrm{L}^{*}{ }_{1}=1,25$; $4-\mathrm{L}^{*}{ }_{1}=1,042 ; 5-\mathrm{L}^{*}{ }_{1}=0,833 ; 6-\mathrm{L}^{*}{ }_{1}=0,625$.

The computational experiments with multichamber ionizing air cleaners (for example, «Super-Plus-Turbo» of the first modification) have shown that selecting a needle electrode in the backward semisphere of this unit has allowed obtaining better results in comparison with other types of electrodes. The example case of calculation data of a plane-parallel electrostatic field in multichamber ionizing air cleaners is shown in Fig. 4.
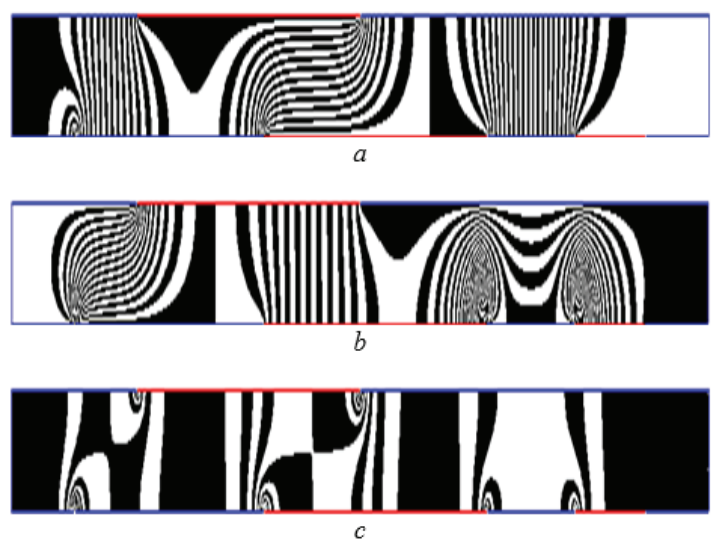

Fig. 4. The standard field calculation data for multichamber ionizing air cleaners: $a$-distribution of the electrostatic field's equipotential surfaces with the given pitch $\Delta \varphi ; b$ - distribution of force surfaces of the field intensity flux with the given pitch $\Delta \chi ; c$ - distribution of electrostatic field's equal intensities surfaces with the given pitch $\Delta E$.

As follows from analyzing the existing models of single-chamber ionizing air cleaners, the corona-forming electrode is placed with regard to grounded plate electrodes. As the calculation data show the design of 
these appliances is not too optimal and efficient, in contrast with the two-stage electrostatic precipitators, for example, EF, EFO, EF/SP, EFO/SP (of companies «PlymoVent» (Sweden) and «SovPlim», St.Petersburg), or electric precipitators of EFVA type (LLC «Elstat», Moscow), which are developing in the right direction. It was found that the development of multichamber ionizing air cleaners' models (e.g., «Super-Plus-Turbo» of the $2^{\text {nd }}$ modification) on the basis of empirical approach resulted in significant deterioration of ionizing air cleaners' properties as compared to the initial model due to installing saw-shaped corona-forming electrodes in the forward semisphere instead of wire electrodes and excluding the needle electrode, as the field intensity in the backward semisphere of the unit decreased by several times as compared to the initial model.

It was established that the mathematical model of a two-stage electrostatic precipitator, as a control object, can be presented as an aperiodic link, while the time constant of the transition process, dealing with the alteration of positive air ions concentration $\left(\mathrm{N}_{+}{ }^{*}\right)$, is within the scope of tens of seconds.

\section{Practical research}

The xperimental research of multichamber ionizing air cleaners with account of the alteration of the type of corona-forming electrodes and their location has shown that that the relative specific concentration of negative air ions $\left(\mathrm{N}_{-}{ }^{*}\right)$ can change by 4 times (air ions meters of 2 types - «Sapfir-3k» and «MAS-01» were used). These results qualitatively coincide with the numerical computation data of the electrostatic field intensity alteration of multichamber ionizing air cleaners for various types of electrodes. It was established that with the increase of distance from air ions sources (ionizing air cleaners), firstly, the average relative specific concentration of both positive and negative air ions at the exit of these units is decreased, and secondly, the average relative velocity of the «ionic wind» is also decreased.

The comparative analysis of the calculation data, of the known and obtained experimental data for the objects under research and other types of electrostatic units has shown that the made assumptions for the calculation of electrostatic units for air filtration and ionization on the basis of second-order elliptical equations with the constant coefficients with account of mixed-type boundary conditions are substantiated. The qualitative and quantitative coincidences of mathematical modeling results of the objects under research with the experimental data and the findings of other authors confirm the validity of the obtained results.

The peculiarities of the general algorithm of numerical study for electrostatic units for air filtration and ionization are presented. Taking into account that in practice it's not always possible to implement the optimality criterion for electrostatic units, so the task of quasioptimal units structure synthesis consists in providing the maximum effective ionization area $\mathrm{F}^{*} \mathrm{e}(\mathrm{m})$ with account of alteration of both the electrodes locations and distances between them, the ratios of chambers' sizes and gaps between them, and the potentials on the electrodes of the researched object at the specified ratios between them. In this regard the problem of determining rational parameters of electrostatic units is also considered, which can be established with the use of the dependences, obtained in the course of work. At this, according to the developed computer modeling methodology, namely, with account of its second stage, the acceptable rational parameters of ionization chamber are established.

As a result of the carried-out research there were presented the peculiarities of structures and circuit designs of automated ventilation systems on base of energy-efficient equipment with the use of two-stage electrostatic precipitator and autonomous ionizing air cleaners with the possibility of ionizing air in the premises of buildings and constructions, with the automated ventilation system being part of automated dispatch control system (ADCS) of the distributed electric power systems of buildings.

\section{Conclusions}

It was established that the mathematical model of a twostage electrostatic precipitator, as a control object, can be presented as an aperiodic link, while the time constant of the transition process, dealing with the alteration of positive air ions concentration $\left(\mathrm{N}_{+}{ }^{*}\right)$, is within the scope of tens of seconds [6].

As a result of the carried-out research the peculiarities of structures and circuit designs of automated ventilation systems on base of energyefficient equipment with the use of two-stage electrostatic precipitator and autonomous ionizing air cleaners are presented.

From analyzing the numerical modeling results of two-stage electrostatic precipitators it was established that the effective ionization area $\left(\mathrm{F}^{*} \mathrm{e}\right)$ can be increased within the scope of $25 \%$ by changing the location of corona-forming electrode and the gap between the chambers, and with the additional account of fringe effects of ionization chamber the maximum effective ionization area $\left(\mathrm{F}^{*}(\mathrm{max})\right)$ increases by 2,5 times.

\section{References}

1. O. Seppanen, The energy-efficient ventilation systems for providing the high-quality indoor climate, V., h., air cond., h. sup. and con. th. ph. (AVOK). 5, 23-28 (2000)

2. S.A. Chistovich, V.K. Averyanov, Y.Y. Tempel, S.I. Bykov, Automated heating and heat supply systems (Stroyizdat, Leningrad, 1987)

3. V.M. Paskonov, V.I. Polezhaev, L.A. Chudov, Numerical modeling of heat and mass exchange processes (Nauka, 1984)

4. I.P. Vereschagin, V.E.Bobrikov, Parameter selection at the electric field computation by the 
equivalent charge method, 1, 38-44 (Electricity, 1988)

5. Y.A. Gol'tsov, A.S. Kizhuk, V.G.Rubanov, Int. J. Pharm. Techn. 12, (2016)

6. A.N. Potapenko, A.V. Belousov, L.I. Koltunov, E.A. Potapenko, News of H. Ed. Inst. Problems of power industry, 5-6, 69-84 (2006) 